Artículo científico

Volumen 32(3):750-763. Septiembre-diciembre, 2021 e-ISSN 2215-3608, doi:10.15517/am.v32i3.44403 https://revistas.ucr.ac.cr/index.php/agromeso/index

\title{
Manejo agronómico sobre el rendimiento y la calidad de café (Coffea arabica) variedad Castillo en Nariño, Colombia ${ }^{1}$
}

\section{Agronomic management on the yield and quality of coffee (Coffea arabica) Castillo variety in Nariño, Colombia}

\author{
Johanna Alixa Muñoz-Belalcazar², Carlos Andrés Benavides-Cardona², Tulio César Lagos-Burbano², Claudia \\ Patricia Criollo-Velázquez $z^{2}$
}

1 Recepción: 2 de noviembre, 2020. Aceptación: 12 de abril, 2021. Este trabajo formó parte del proyecto "Investigación evaluación del efecto de sombra de diferentes especies arbóreas en el comportamiento agronómico y calidad de Café, Consacá, Nariño, Occidente", financiado por el Sistema General de Regalías y ejecutado por la Universidad de Nariño.

2 Universidad de Nariño, sede VIPRI Pasto, Grupo de Investigación en Producción de Frutales Andinos, 520001, Nariño, Colombia. mjohannaalixa@gmail.com (autor para la correspondencia; https://orcid.org/0000-0003-4466-192X); cabenavides@udenar.edu.co (https:// orcid.org/0000-0002-9144-6168); tclagosb@udenar.edu.co (https://orcid.org/0000-0001-9222-4674); ingcrivel@gmail.com (https://orcid. org/0000-0003-0763-1115).

\section{Resumen}

Introducción. En los últimos años el área y la producción de café ha incrementado en Nariño, pero su sostenibilidad y rentabilidad se afectó por diversos factores. El aprovechamiento de atributos de calidad podría ser una alternativa para mejorar la actividad económica. Objetivo. Analizar la incidencia del manejo agronómico del sistema productivo de café variedad Castillo, sobre el rendimiento y la calidad del grano en el departamento de Nariño. Materiales y métodos. El trabajo se desarrolló durante 2018-2019 en cuatro municipios del departamento de Nariño, Colombia. Con sesenta unidades productivas (UPC) con café variedad Castillo. Se diseñó un instrumento mixto para la captura de información en predios y de variables in situ sobre el rendimiento y la calidad, con protocolos de la Specialty Coffee Association of America (SCAA) y la Federación Nacional de Cafeteros (FNC). Resultados. El 41,6 \% de las UPC tenían predios sobre $1700 \mathrm{msnm}$, el 46,6\% se asoció con especies forestales, el 33,3\% con frutales o musáceas y el $20 \%$ a libre exposición. El histograma de valores propios, permitió seleccionar cinco factores que explicaron el 52,69 \% de variabilidad, las variables contribuyentes se relacionaron con: tipo de fertilizante, frecuencia, dosis de abono orgánico y densidad. El análisis de clasificación permitió conformar cuatro grupos, el cuarto con mayor número de UPC (35) pertenecía a la zona occidente, empleaba densidades de siembra >5500 plantas/hectárea y combinaba fertilizantes químicos y orgánicos. Conclusiones. Los sistemas mixtos con café bajo sombrío prevalecieron en su mayoría para el sistema productivo estudiado, de tal manera que no derivaron en composición de grupos diferenciales, como si hubo con la variabilidad en densidades de siembra, uso de abono orgánico y fraccionamiento de la fertilización. Estas variables de manejo agronómico influyeron en la obtención de diferencias para el rendimiento y la calidad de grano comercializable.

Palabras clave: sistema agroforestal, factor de rendimiento, caficultura. 


\begin{abstract}
Introduction. In recent years the area and production of coffee has increased in Nariño, but its sustainability and profitability have been affected by various factors. Taking advantage of quality attributes could be an alternative to improve the economic activity. Objective. To analyze the incidence of agronomic management of the Castillo variety coffee production system on the yield and quality of the bean in the Nariño department. Materials and methods. The work was developed during 2018-2019 in four municipalities of the department of Nariño, Colombia. With sixty production units (UPC) with Castillo variety coffee. A mixed instrument was designed to capture information in farms and in situ variables on yield and quality, using protocols of the Specialty Coffee Association of America (SCAA) and the Federacion Nacional de Cafeteros (FNC). Results. $41.6 \%$ of the UPCs had farms above 1700 meters above sea level, $46.6 \%$ were associated with forest species, $33.3 \%$ with fruit trees or musaceae and $20 \%$ with free exposure. The histogram of eigenvalues, allowed the selection of five factors that explained the $52.69 \%$ of variability, the contributing variables were related to: type of fertilizer, frequency, dose of organic fertilizer, and density. The classification analysis allowed the formation of four groups, the fourth with the highest number of UPC (35) belonged to the western zone, used planting densities $>5500$ plants/hectare and combined chemical and organic fertilizers. Conclusions. Mixed systems with coffee under shade prevailed in their majority for the productive system studied, in such a way that they did not derive in the composition of differential groups, as there was with the variability in planting densities, use of organic fertilizer and fractionation of fertilization. These agronomic management variables influenced the obtaining of differences for the yield and marketable bean quality.
\end{abstract}

Keywords: agroforestry system, yield factor, coffee-culture.

\title{
Introducción
}

En muchos países en desarrollo, la producción de café (Coffea arabica) se considera como una actividad de impacto tanto en el ámbito social como en el económico, que genera como producto de exportación cuantiosas divisas que hacen que este sector no solo contribuya en la economía de los países exportadores, sino también de los importadores y proporciona un medio de vida para más de veinticinco millones de agricultores y sus familias, en su mayoría con producción en pequeña escala (Maurice \& Davis, 2011; McNougher, 2013; Panhuysen \& Pierrot, 2014; Sánchez, 2015; Valencia, 2016).

En las dos últimas décadas la producción de café aumentó en un $50 \%$, a pesar del crecimiento general del sector, los precios tuvieron una tendencia continua al descenso, debido al incremento en la producción, depreciación de la moneda local de algunos países productores frente al dólar estadounidense, aumento de la competitividad de algunos países en el mercado mundial, especulación en los mercados futuros y a que las tendencias a largo plazo de los precios del café en algunos países han sido negativas (Organización Internacional del Café, 2019).

La producción de café en Colombia presentó un crecimiento cercano al $90 \%$ en los últimos años, al pasar de 7,7 millones en 2012 a 14,7 millones de sacos de 60 kg de café verde en 2019 (Federación Nacional de Cafeteros de Colombia, 2020). El cultivo de café en el país ha sido establecido bajo diversos sistemas de producción, tales como el monocultivo bajo sombra con perennes como musáceas o en arreglos con diferentes especies leñosas como forestales y frutales (Alvarado et al., 2013). La práctica de los sistemas del cultivo de café en asocio con forestales ha tomado un gran interés y, por ende, se promueve su adopción no solo para el mejoramiento de las condiciones microclimáticas, sino también por su acción positiva en la calidad del grano (Jezeer \& Verweij, 2015; Valencia, 2016).

El departamento de Nariño participa con el 4,5 \% en la producción nacional de café (Federación Nacional de Cafeteros de Colombia, 2018; Ministerio de Agricultura y Desarrollo Rural [MADR], 2018). Además, debido a su 
posición geográfica se caracteriza por incluir también la producción de "cafés de altura", debido a que es posible encontrar cultivos en altitudes hasta los 2200 msnm (Puerta-Quintero, 2016; Ramírez et al., 2013). Estos y otros atributos asociados a sus sistemas de producción y al origen volcánico de los suelos, han permitido que el café de Nariño haya sido catalogado por algunos expertos en diferentes ocasiones como uno de los mejores del país, destacándose a nivel nacional e internacional por sus características diferenciadoras en sabor y aroma (Burgos, 2015; De la Rosa, 2011).

En Colombia, el mercado desde la perspectiva de café tradicional ha presentado dificultades, a pesar de que su producción y consumo, tanto para el ámbito local como para su exportación, han crecido pero sus precios han disminuido (Echavarría et al., 2015; Velásquez \& Trávez, 2019). Una posible solución identificada por estos mismos autores, señala la necesidad de realizar una transición a cultivos con fines de producción de cafés denominados como especiales. Esto, con base en la historia que el país tiene en el sector y a las condiciones geográficas con las que cuenta.

El término "café especial", hace referencia a la conjunción de factores de tipo geográfico y productivo que permiten la obtención de granos de café con sabor único y de características particulares que preservan su identidad (Ponte, 2004) y según La Asociación Americana de Café Especial (SCAA), lo define como aquellos cafés que conservan una consistencia en sus características físicas (forma, tamaño, humedad, apariencia y defectos), sensoriales (olfativas, visuales y gustativas), prácticas culturales (recolección, lavado, secado) y en sus procesos finales (tostión, molienda y preparación); características que los distinguen del común de los cafés y brindan al consumidor una experiencia diferencial, basada en una bebida de mayor calidad en un mercado en donde el producto se encuentra homogenizado y por las cuales los clientes están dispuestos a pagar un precio superior, lo cual también repercute en el bienestar del productor (Giovannucci \& Koekoek, 2003; Specialty Coffee Association of America, 2004).

Diversos autores, han estudiado aspectos diferenciales en manejo agronómico, condiciones ambientales, sistemas de producción, manejo de cosecha y poscosecha, entre otros, sobre la determinación de sus efectos en el rendimiento y calidad del grano (Arcila et al., 2007; Aristizábal \& Duque, 2006; Joët et al., 2010; Montilla et al., 2008; Orozco et al., 2011), pero aún se desconoce con exactitud los factores que influyen en la permanencia desde este punto de vista en la calidad del grano, esto debido posiblemente a la diversidad de ambientes en los que se produce el cultivo de café. Otros lo atribuyen de forma general a la obtención de granos de calidad a la exposición de factores climáticos como la altitud de producción, condiciones agroecológicas del cultivo, variedad sembrada, densidad de siembra, condiciones fitosanitarias, nutrición del cultivo, proceso de beneficio, y pos cosecha, los cuales puede influir significativamente en la composición bioquímica, calidad física y organoléptica de la taza de café (Aristizábal \& Duque, 2006; Bertrand et al., 2006; Buenaventura \& Castaño, 2002; Lara \& Vaast, 2007). Por ejemplo, Suárez et al. (2015) encontraron que al disminuir la temperatura por efectos de la altitud, se prolonga el proceso de maduración de la cereza, lo que favorece el llenado, peso de grano y mayor calidad de taza, como resultado de la lenta maduración del grano, además, de ser un factor reconocido en los mercados internacionales. Esta situación, no ha permitido establecer con claridad requerimientos y modelos estandarizados que permitan alcanzar en forma continua, niveles de calidad que puedan ser sostenibles en el tiempo y lograr así estándares requeridos por los mercados especializados de manera estable (Avelino et al., 2005; Banegas, 2009; DaMatta et al., 2007; Geromel et al., 2007).

El presente trabajo se planteó como objetivo analizar la incidencia del manejo agronómico del sistema productivo café variedad Castillo, sobre el rendimiento y la calidad del grano en el departamento de Nariño. 


\section{Materiales y métodos}

\section{Localización}

La investigación se llevó a cabo durante los años 2018 - 2019, periodo que comprendió las fases de desarrollo de inflorescencias, desarrollo de botones florales, floración, crecimiento del fruto, maduración, cosecha y postcosecha del grano, en los municipios cafeteros de La Unión, Sandoná, La Florida y Consacá del departamento de Nariño. En cada municipio se estudiaron los sistemas productivos de café (Coffea arabica), localizados en tres diferentes rangos altitudinales de $\leq$ a $1500 \mathrm{msnm}$, entre 1501 y $1700 \mathrm{msnm}$ y por encima de $1700 \mathrm{msnm}$. El municipio de la Unión se encuentra ubicado en el ecotopo cafetero 220A, su ubicación geográfica de referencia se encuentra entre $1^{\circ} 21^{\prime \prime}-1^{\circ} 42^{\prime \prime}$ LN y hace parte de la cuenca del río Patía y los municipios de Sandoná, La Florida y Consacá hacen parte del ecotopo cafetero 221A, su ubicación geográfica se encuentra entre $1^{\circ} 05^{\prime \prime}-1^{\circ} 36^{\prime}$ LN y forma parte de la cuenca del río Guitará (Gómez et al., 1991). En las zonas de estudio, el manejo del cultivo se realizaba en forma tradicional y está relacionado con la cultura y la economía familiar (Burgos, 2015).

\section{Población y obtención de la información}

En tres localidades representativas de la zona productiva de café en el departamento de Nariño, se seleccionaron 82 unidades productivas (UPC), que eran fincas o parcelas, donde se llevaban a cabo actividades de producción con café, de entre tres y cinco años de establecido con la variedad registrada Castillo y ubicadas en tres altitudes. De estas, mediante la aplicación de un proceso de muestreo no probabilístico denominado por cuotas (Otzen \& Manterola, 2017), se seleccionaron sesenta productores tipo, como representativos de los municipios en estudio; dieciocho en el municipio de La Unión, y catorce en cada uno de los municipios de Sandoná. La Florida y Consacá como base de cuota.

En cada UPC, para la obtención de la información, se aplicó un formulario mixto tipo encuesta y captura de información directa, fue estructurado con 33 variables relacionadas con su identificación, características de manejo agronómico, producción y calidad de grano obtenido.

La aplicación del formulario, se realizó mediante entrevista personal y observación directa de las UPC. Con el fin de tener un conocimiento más detallado y real de los sistemas productivos del cultivo de café, las variables relacionadas con la producción fueron registradas en los lotes de observación y las variables de calidad, determinadas por el laboratorio de la Asociación Gestores Profesionales del Sur (GPS).

\section{Variables evaluadas}

Mediante visitas realizadas a las UPC seleccionadas y con base en la información entregada por el propietario o administrador de la finca, se registraron en primer lugar los datos de pasaporte y entre las variables relacionadas con el manejo agronómico del cultivo: edad, uso de árboles para sombrío, especies utilizadas en el sombrío, densidad de siembra, aplicación de riego, tipo de riego, dosis y frecuencia de fertilización, manejo de arvenses, presencia y control de problemas fitosanitarios.

Las variables de producción como rendimiento por árbol de café cereza, relación café cereza pergamino seco (CPS), producción en arrobas (una arroba $=12,5 \mathrm{~kg}$ de CPS), se registraron en cada localidad experimental. Previo a la cosecha se evaluó el rendimiento por árbol en una muestra aleatoria de quince plantas por unidad estudiada. En estas, para la estimación de producción de café cereza, se contó el número total de frutos por planta y el peso de fruto que fue calculado a partir del promedio de cuatro muestras de cien frutos tomadas al azar en cada lote evaluado. 
La evaluación de la relación café cereza: CPS, se determinó a partir de diferenciales de peso en una muestra de 1 $\mathrm{kg}$ de café cereza tomado por lote experimental, que fue sometido a un proceso de beneficio tradicional (se secó a libre exposición solar, se registró el peso cuando las muestras alcanzaron un grado de humedad entre 10 - $12 \%$ ). A partir de los datos obtenidos, también se estimó la producción de CPS en arrobas por hectárea.

Dentro de las variables de calidad del grano se consideraron: a) el factor de rendimiento, establecido como la cantidad de café pergamino seco que se necesita trillar para obtener un saco de $70 \mathrm{~kg}$ de café excelso de exportación (Puerta-Quintero, 2016) y clasificado por la Federación Nacional de Cafeteros de Colombia (2020) como factor $<94$ mayor calidad, igual a 94 precio base y >94 menor calidad y b) puntaje final de taza, dado por el catador y determinado por la suma de todos los atributos del análisis sensorial (aroma, sabor, sabor residual, acidez, cuerpo, uniformidad, balance, taza limpia y dulzor), para esto, la Asociación de Cafés Especiales de América (SCAA, por sus siglas en inglés) (Specialty Coffee Association of America [SCAA], 2015), ha establecido una clasificación con base en el puntaje obtenido: $<80$ por debajo del café especial, entre 80-84,99 muy bueno, entre 85-89,99 excelente y de 90-100 excepcional.

Las variables relacionadas con el análisis físico y sensorial (proceso de catación) se determinaron según las normas y procedimientos del laboratorio AGPS en el municipio de La Unión Nariño, el cual maneja los protocolos estándares de la SCAA (2015) y las normas de la Federación Nacional de Cafeteros de Colombia, laboratorio que cuenta con personas certificadas por el Instituto de Calidad de Café (CQI, por sus siglas en inglés).

\section{Análisis estadístico}

Todas las respuestas (33) del cuestionario se categorizaron, según rangos representativos determinados para el propósito de este estudio y a no más de tres categorías por variable, lo cual permitió mantener la información intacta respecto a las unidades de observación, esto con el fin de facilitar la aplicación del método multivariado de análisis de correspondencias múltiples (ACM) y el análisis de clasificación jerárquica, con base en las distancias de Ward, con el software SPAD 5.6 (Chávez et al., 2010).

\section{Resultados}

\section{Análisis descriptivo y correspondencias múltiples}

Una vez aplicado el instrumento para consolidación de la información, se estableció que a nivel geográfico el $10 \%$ de las UPC analizadas, de acuerdo con el análisis descriptivo se encuentran en altitudes de $\leq 1500 \mathrm{msnm}$, el 48,3\% entre $1501 \mathrm{msnm}$ y $1700 \mathrm{msnm}$ y el $41,6 \%$ a más de $1700 \mathrm{msnm}$. De estas, el 46,6 \% de los cultivos de café se enccontraban establecidos bajo un contexto de siembra en asociación con forestales, el $33,3 \%$ con frutales o musáceas y $20 \%$ a libre exposición solar.

En cuanto a las localidades analizadas, del total de los sistemas productivos asociados con forestales, el 55,6 $\%$ se encontraba en el municipio de la Unión y el restante se distribuyó en las demás localidades del presente estudio. Además, esta condición resulta relevante para la observación del factor de rendimiento ya que, de las UPC con arreglos agroforestales, el $75 \%$ obtuvo un factor $\leq 90$, y el $15 \%$ entre 85 y 89,99 versus, los sistemas a libre exposición en los cuales se observó que el $66,7 \%$ obtuvieron un factor $\leq 90$.

Con respecto a los sistemas productivos mencionados, la densidad de siembra predominante para el sistema productivo de café variedad Castillo (83,3\%), independiente de la zona de producción o de la altitud, fue de más de 5500 plantas por hectárea, con diferentes arreglos distanciales respecto a las asociaciones mencionadas (Cuadro 1). Caso contrario ocurrió con la incidencia del problema sanitario de mayor interés identificado por los productores 
Cuadro 1. Densidad de siembra y afectación por broca del café (Hypotenemus hampei) en sistemas productivos de café (Coffea arabica) variedad Castillo en la zona productora del departamento de Nariño, Colombia, Universidad de Nariño. 2018-2019.

Table 1. Planting density and coffee berry borer (Hypotenemus hampei) affectation in Castillo variety coffee (Coffea arabica) production systems in the production zone of the department of Nariño, Colombia, University of Nariño. 2018-2019.

\begin{tabular}{lccc}
\hline Densidad de siembra & UPC $*$ & Afectación Broca café & UPC \\
\hline Plantas/hectárea & $\%$ & Altitud $(\mathrm{msnm})$ & $\%$ \\
Menos de 4500 & 10 & Hasta 1500 & 83,33 \\
$4501-5500$ & 6,7 & $1501-1700$ & 75,99 \\
Más de 5500 & 83,3 & Más de 1700 & 28 \\
\hline
\end{tabular}

*UPC: unidades productivas / productive units.

broca del café (Hypotenemus hampei). El cual, se vio afectado por la altitud, de tal manera que de las UPC ubicadas en el rango de entre 1500 a $1700 \mathrm{msnm}$, el $83 \%$ de los sistemas mostró presencia de la plaga y de las UPC ubicadas por encima de $1700 \mathrm{msnm}$, el 75,9\% evidenció este problema. El 67,6\% de los lotes que presentaron afectación por broca tuvieron un factor de rendimiento $<90$ frente al 76,9\% de los que no presentaron la plaga, al relacionar este aspecto con el puntaje en catación para calidad de taza solo un 5,9\% de los lotes afectados obtuvieron un puntaje de taza de catación entre 85 y 89,99 café de excelente calidad.

De los productores encuestados, cabe mencionar que el $80 \%$ empleó una fuente orgánica en mezcla con un fertilizante químico a la hora de la siembra, mientras que el $90 \%$ en etapa de producción solo empleó fertilizante químico.

Los mejores factores de rendimiento ( $\leq 90)$ y puntajes finales de catación (80 - 85) se observaron en los rangos altitudinales entre $1501-1700 \mathrm{msnm}$ con un $65,5 \%$ y $72,4 \%$, respectivamente.

Con respecto a los parámetros de nutrición se resaltaron dos tendencias principales, fertilización fraccionada en dos partes en el año y una sola aplicación durante el mismo periodo. En la primera el $37 \%$ de los productores obtuvieron más de 250 arrobas de CPS por hectárea, mientras que de los que lo hacen una vez al año, el $100 \%$ alcanzaron producciones $\leq 150$ arrobas de café CPS por hectárea.

En lo relacionado con el puntaje final de tasa el $11 \%$ de los productores que fraccionan la fertilización, obtuvo entre 85 y 89,99 puntos, contrario a quienes realizaron la práctica una vez al año, quienes en ningún caso alcanzaron este rango. La mayoría de productores $(86,6 \%$ ), aplicaron una dosis $>100 \mathrm{~g}$ de fertilizante por planta, de ellos, el 36,5\% con producciones de $>250$ arrobas de CPS por hectárea, se determinó además, que de los productores que aplicaron de 50 a $100 \mathrm{~g}$ por planta solo el 12,5\% obtuvieron producciones de este nivel. Finalmente, cabe mencionar que el $30 \%$ de las UPC obtuvieron más de $3 \mathrm{~kg}$ de café cereza por planta, el $33 \%$ obtuvo más de 250 arrobas CPS ha-1 ${ }^{-1}$ el 71,66 \% presento un factor de rendimiento menor a 90 y en igual porcentaje de UPC obtuvieron un puntaje de catación entre 80 - 84,99 catalogado como café de muy buena calidad (Cuadro 2).

El análisis del histograma de valores propios (Cuadro 3), permitió seleccionar de entre 35, los primeros cinco factores que explicaron en conjunto el $52,69 \%$ de la variabilidad total, que si bien es baja se considera adecuada puesto que desde el sexto factor en adelante la acumulación porcentual fue mínima, lo que evidenció rangos porcentuales desde $0,2 \%$ hasta el $2 \%$. La variabilidad seleccionada, está relacionada con variables de tipo cualitativo que permitieron inferir acciones de manejo agronómico sobre aspectos de rendimiento y calidad del café variedad Castillo.

A partir del análisis de correspondencias múltiples (Cuadro 4), se establecieron las variables que más contribuyeron a la conformación de los factores, de tal manera que para el caso del primero se encontraron: tipo 
Cuadro 2. Parámetros de rendimiento y calidad en sistemas productivos de café (Coffea arabica) variedad Castillo en tres zonas altitudinales de producción del departamento de Nariño-Colombia, Universidad de Nariño, periodo 2018-2019.

Table 2. Yield and quality parameters in Castillo variety coffee (Coffea arabica) production systems in three altitudinal production zones in the department of Nariño-Colombia, University of Nariño, period 2018-2019.

\begin{tabular}{|c|c|c|c|}
\hline \multicolumn{4}{|c|}{ Parámetros de rendimiento } \\
\hline kg plant $^{-1}$ (cereza) & $\%$ UPC & Arrobas* $\mathrm{CPS} \mathrm{ha}^{-1}$ & $\%$ UPC \\
\hline Menor a 2 & 37 & Menor a 150 & 30 \\
\hline 2 a 3 & 33,3 & 150 a 250 & 36,6 \\
\hline Mayor de 3 & 30 & mayor de 250 & 33 \\
\hline \multicolumn{4}{|c|}{ Calidad de grano } \\
\hline Factor de rendimiento & $\%$ UPC & Puntaje catador & $\%$ UPC \\
\hline Menor a 90 & 71,66 & Menor a 80 & 18,83 \\
\hline 90 a 94 & 16,6 & 80 a 85 & 71,66 \\
\hline Mayor de 94 & 11,6 & 85 a 89,9 & 9,5 \\
\hline
\end{tabular}

* Una arroba $=12,5 \mathrm{~kg}$ de café cereza pergamino seco (CPS) / One at sign $=12,5 \mathrm{~kg}$ of dry parchment cherry coffee (CPS).

Cuadro 3. Representación porcentual de la variabilidad observada en las variables cualitativas relacionadas con la incidencia del manejo agronómico del cultivo de café (Coffea arabica) variedad Castillo con la producción y la calidad de taza en cuatro municipios de Nariño, Colombia, Universidad de Nariño. 2018-2019.

Table 3. Percentage representation of the observed variability in the qualitative variables related to the incidence of the agronomic management of the Castillo variety coffee (Coffea arabica) cultivation with production and cup quality in four municipalities in Nariño, Colombia, University of Nariño. 2018-2019.

\begin{tabular}{ccccc}
\hline Factor & Valor Propio & Porcentaje & Porcentaje acumulado & Histograma \\
\hline 1 & 0,3001 & 18,01 & 18,01 & $* * * * * * * * * * * * * * * * *$ \\
2 & 0,1800 & 10,80 & 28,80 & $* * * * * * * * * *$ \\
3 & 0,1545 & 9,27 & 38,07 & $* * * * * * * * *$ \\
4 & 0,1305 & 7,83 & 45,90 & $* * * * * * *$ \\
5 & 0,1130 & 6,78 & 52,69 & $* * * * *$ \\
\hline
\end{tabular}

de fertilizante empleado en siembra y producción, frecuencia de aplicación de abono orgánico al cultivo, dosis de abono orgánico aplicado en producción y densidad de siembra por hectárea. Para el factor dos, altitud, incidencia y prácticas de manejo en broca y otras plagas en el cultivo de café se evidenciaron como las variables de mayor contribución.

En los últimos tres factores, las variables que hicieron el mayor aporte fueron: incidencia de los vientos y tipo de control de plagas para el tercero, aplicación de riego y puntaje en calidad de taza para el cuarto y densidad de siembra por hectárea, frecuencia en aplicación de fertilizante químico y factor de rendimiento para el quinto. Este último, con una contribución débil del 6,7 \% que podría referir a una incidencia débil en el comportamiento general del sistema productivo en una de las variables de respuesta representativa como lo es el factor de rendimiento. Sin embargo, la inclusión del puntaje en el factor 4 podría en cierta medida establecer influencias de manejo en este aspecto cuando se refiera el tema de productividad. 
Cuadro 4. Medidas de discriminación de las variables que más contribuyeron a los factores principales, sobre la incidencia del manejo agronómico del sistema productivo de café (Coffea arabica) variedad Castillo sobre el rendimiento y calidad del grano en Nariño, Colombia. Universidad de Nariño. 2018-2019.

Table 4. Measures of discrimination of the variables that most contributed the most to the principal factors on the incidence of agronomic management of the Castillo coffee (Coffea arabica) variety production system on yield and grain quality in Nariño, Colombia. University of Nariño. 2018-2019.

\begin{tabular}{lccccc}
\hline & \multicolumn{5}{c}{ Factores } \\
\cline { 2 - 6 } Variables & $\mathbf{1}$ & $\mathbf{2}$ & $\mathbf{3}$ & $\mathbf{4}$ & $\mathbf{5}$ \\
\hline Altitud & 0,4 & 12,7 & 8,8 & 5,4 & 5,6 \\
Aplicación de riego al cultivo & 0,8 & 0,3 & 1,5 & 20,9 & 5 \\
Incidencia de los vientos & 5,9 & 6,0 & 15,6 & 0,9 & 6,2 \\
Tipo de abono utilizado en la siembra & 12,5 & 0,9 & 5,5 & 9,3 & 2,1 \\
Tipo de abono utilizado en producción & 10,0 & 0,0 & 0,0 & 0,0 & 0,1 \\
Frecuencia de aplicación de abono orgánico & 11,3 & 1,1 & 0,0 & 0,4 & 2,8 \\
Dosis de abono orgánico aplicado en producción & 11,3 & 1,4 & 2,7 & 1,0 & 3.2 \\
Frecuencia de aplicación de fertilizante químico & 8,3 & 4,9 & 0,6 & 6,0 & 14,2 \\
Incidencia de broca en el cultivo & 0,6 & 11,6 & 7,9 & 0,4 & 2,0 \\
Tipo de control de broca & 0,4 & 11,5 & 8,5 & 0,6 & 2,7 \\
Tipo de control de plagas en el cultivo & 3,1 & 10,6 & 10,0 & 1,2 & 0,8 \\
Densidad de siembra por hectárea & 13,5 & 4,0 & 1,8 & 2,6 & 11,0 \\
Factor de rendimiento & 1,9 & 1,2 & 0,8 & 0,5 & 9,4 \\
Puntaje de calidad de taza & 1,0 & 0,8 & 0,7 & 19,7 & 4,7 \\
\hline
\end{tabular}

\section{Análisis de clasificación}

El análisis de clasificación, permitió conformar cuatro grupos bien definidos. Los individuos agrupados en cada clase se observan en la Figura 1. El primero, conformado por ocho productores que representaron el 13,33 $\%$ del total, se caracterizó porque, de ellos, el 87,5 \% utilizaba abono orgánico al momento de la siembra, el 75 $\%$ fertilizaron con una dosis de 50 a 100 g por planta dos veces al año, el $100 \%$ obtiene una relación café-cereza: CPS $>5$ y el $100 \%$ de los productores de este grupo utilizaba densidades de siembra entre 4501 y 5500 plantas por hectárea.

El grupo 2 se conformó por tres individuos (5\%) y se definió porque el $100 \%$ de los cultivos de las UPC que lo representan, con densidades de siembra $\leq 4500 \mathrm{pl} \mathrm{ha}^{-1}$; los tres productores aplicaban abono orgánico una vez al año intercalándolo también con el fertilizante químico en la etapa de producción. Además, dos de los productores solo realizaban una aplicación de fertilizante por año.

El grupo 3 está conformado por catorce productores de café, que representaban el 23,33\% de la muestra. Las UPC de este grupo se caracterizaron porque el 92,86 \% pertenecía al municipio de La Unión, el $100 \%$ de las fincas presentaron vientos leves, el 78,57 \% obtuvieron una relación café-cereza CPS de $\leq 5$ y el 78,57 \% realizan control químico de plagas.

El cuarto grupo estuvo conformado por 35 unidades productivas, las cuales representaron el $58,33 \%$ de la muestra de estudio y se caracterizó porque el $100 \%$ de las UPC correspondieron a la zona occidente del departamento de Nariño, manejaban densidades de siembra de >5500 plantas por hectárea, utilizaban abono orgánico y químico al momento de la siembra, aplicaban abono químico en la etapa de producción, utilizaban una dosis de fertilizante de $>100$ g por planta, fertilizaban dos veces al año y no aplicaban abono orgánico en producción. 


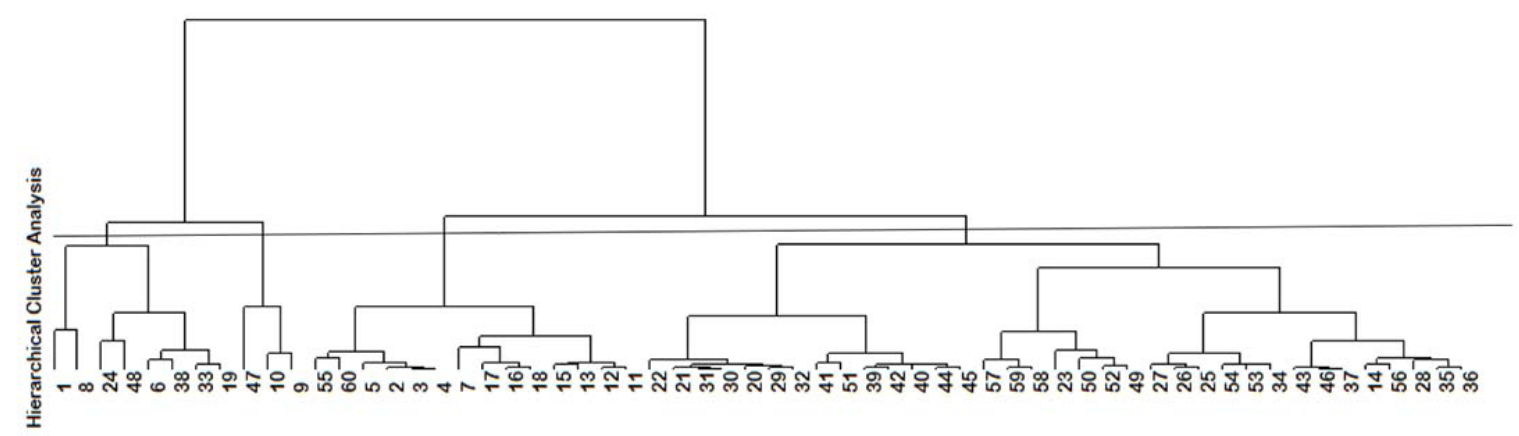

Figura 1. Grupos conformados por unidades productivas de café (Coffea arabica) variedad Castillo en cuatro municipios del departamento de Nariño, Colombia. Universidad de Nariño. 2018-2019.

Figure 1. Groups made up of Castillo variety coffee (Coffea arabica) production units in four municipalities of the department of Nariño, Colombia. University of Nariño. 2018-2019.

\section{Discusión}

La zona sur de Colombia, en donde se enmarcan las regiones productoras de café del departamento de Nariño, los sistemas productivos, se caracterizan por encontrarse en gran proporción en zonas altitudinales altas (Gamboa et al., 2015) (variable que aportó en la conformación de los factores 2 y 3 del ACM), por lo que, además, de ser denominado café de altura, su manejo como sistema requiere aspectos diferenciales en cuanto a sistemas tradicionales, tanto para factores de planificación del cultivo como para aspectos de manejo agronómico que influyen en características atribuibles a este tipo de café, como una mayor acidez y calidad de taza, como lo afirmaron también Ramos \& Criollo (2017) y Viloria (2007), quienes señalaron que la mayor parte de los cultivos de café en el departamento de Nariño, se encuentran en pequeños predios o minifundios localizados en alturas superiores a $1700 \mathrm{msnm}$.

Los sistemas que para el presente estudio fueron identificados en las UPC analizadas, correspondieron en su gran mayoría a asociaciones con otras especies, principalmente de tipo forestal y especies frutícolas, bajo diferentes arreglos espaciales. Esta característica, de acuerdo con autores como Alvarado et al. (2013), es congruente con la diversidad de sistemas de producción empleados, no solo en el departamento de Nariño sino en la caficultura colombiana, que si bien para el mismo autor infiere en las características productivas y de calidad de grano, no permiten por su prevalencia definir diferenciales asociados a este aspecto en el presente trabajo. Los mismos autores, también sugirieron diversidad en los aspectos de manejo agronómico y demás actividades realizadas en cada sistema con las variantes adaptadas a los sistemas de producción.

La gestión del ambiente se puede considerar como un factor determinante para la obtención de condiciones diferentes en cuanto a la calidad de los productos cosechados se refiere. Esta situación se observó cuando parámetros como factor de rendimiento ( $\leq 90)$ y rangos en puntajes de calidad de taza $(80-84,99)$, se evidenciaron en mejor manera para altitudes entre 1501-1700 msnm. Sin embargo, además, del efecto ambiental sobre estas propuestas, cabe también mencionar la respuesta fitosanitaria variable en cuanto a condiciones ambientales.

Este factor se observó en el presente trabajo, ya que la presencia de broca del café (Hypothenemus hampei), como uno de los problemas identificados como más limitantes en la UPC, varió con respecto a las zonas altitudinales. Estas condiciones por ende, se asocian con actividades de gestión diferencial al sistema productivo, ya que los problemas fitosanitarios inciden en la calidad del grano, debido a que daña su apariencia y color, además, de obtener una taza de café con sabor y aroma desagradable, un amargo muy fuerte y cuerpo pesado (Bosselman 
et al., 2009; Díaz et al., 2014; Montoya, 1999; Soto et al., 2002), situación identificada en la prueba de catación en cafés de las unidades objeto del estudio.

De acuerdo con diversos autores, las prácticas agronómicas asociadas a la planificación y ejecución de acciones tendientes a la nutrición de cultivos, son determinantes no solo del volumen de producción, sino también en la determinación del perfil de grano en café, por ejemplo cuando se realizan altas aplicaciones de nitrógeno, el grano presenta menor densidad y calidad, la deficiencia de fósforo produce grano pequeño, la de hierro ocasiona granos ámbar pálido, café tostado suave y falta de acidez, la de boro por su parte, produce vaneamiento de granos, los excesos de calcio y potasio producen bebida amarga y áspera, la deficiencia de zinc reduce el tamaño y densidad del grano, la deficiencia de magnesio causa granos marrones y características irregulares en el proceso de tostado (Lara \& Vaast, 2007; Pérez et al., 2005; Puerta, 2001; Rosas et al., 2008).

Si bien, en el presente estudio no se ahondó sobre minerales específicos aplicados, se consideró como aspecto relevante la cantidad, frecuencia y fuente mineral aplicada, razón por la cual se pudo evidenciar que en las UPC, de acuerdo con el manejo dado a este factor, cuando se aplicó mayor dosis y en forma fraccionada se obtuvo no solo mayor rendimiento, sino también mayor calidad de taza, motivo que sugiere la relación positiva entre manejo nutricional y parámetros de productividad en café variedad Castillo.

En general, los resultados tanto de la descripción cualitativa de las UPC, como de los análisis de clasificación y de correspondencias múltiples evidenciados en el presente trabajo, coincidieron con trabajos reportados por otros autores (Arcila et al., 2007; Aristizábal \& Duque, 2006; Joët et al., 2010; Montilla et al., 2008; Orozco et al., 2011), quienes afirmaron, en forma general, que la calidad del grano depende de un conjunto de variables que hacen parte de un sistema muy complejo con variables que aportan en diferente grado a este componente. De tal manera que no es suficiente considerar solo unos factores, hay que tomar en cuenta la interacción entre todas las variables y otros que afectan directamente la calidad como cosecha y pos cosecha.

De igual manera, Puerta-Quintero (2016) confirmó que la calidad de la bebida está determinada por las características físicas del grano, la humedad y los defectos. En 216 fincas muestreadas y localizadas en diferentes altitudes (1050 - $2050 \mathrm{msnm}$ ), 15 unidades de suelo, 112 veredas, 35 municipios y siete departamentos, se encontró café de buena y de mala calidad, lo que evidenció así que la interacción de prácticas agronómicas particulares con la gestión ambiental influyen en forma directa sobre los componentes de rendimiento y calidad del grano de café.

\section{Conclusiones}

El mayor porcentaje de las UPC analizadas, se encontraban establecidas bajo condiciones edafoclimáticas que los catalogan como café de altura. La gestión agronómica de planificación ambiental y de prácticas a realizar, derivaron en diferenciales en cuanto a parámetros productivos y de calidad del café variedad Castillo.

Del análisis cualitativo, se pudo determinar que prevalecieron en la zona estudiada los sistemas de producción mixtos (sistemas bajo sombra con frutales y agroforestales con perennes), aspectos que por su prevalencia se consideran comunes para el sistema productivo en la zona estudiada y por ende, no determinantes de grupos diferenciales, que se puedan considerar como influyentes de la calidad del grano asociados a este factor.

Además, de la altitud como factor relevante, aspectos relacionados con el manejo agronómico del sistema productivo tales como manejo fitosanitario (para broca del café), aplicación de riego, fraccionamiento de fertilización, fuentes y dosis de fertilización, permitieron evidenciar diferenciales en cuanto a parámetros de calidad y factor de rendimiento como independientes, lo que derivó en grupos diferenciales para sistemas en las zonas productoras.

Se identificaron cuatro grupos específicos de productores con características diferenciales en cuanto a manejo agronómico. En estos, se resaltan las UPC del grupo cuatro, que además de su ubicación geográfica, establecieron prácticas como incremento de la biodiversidad en sus sistemas y fraccionamiento de la fertilización, acciones que resultan favorables en el incremento del factor de rendimiento y calidad de grano. 


\section{Agradecimientos}

Los autores, agradecen al Grupo de Investigación en Producción de Frutales Andinos de la Universidad de Nariño y al proyecto "Investigación evaluación del efecto de sombra de diferentes especies arbóreas en el comportamiento agronómico y calidad de café, Consacá, Nariño, Occidente”, financiado por el Sistema General de Regalías (SGR).

\section{Referencias}

Alvarado, J., Andrade, H., \& Segura, M. (2013). Almacenamiento de carbono orgánico en suelos en sistemas de producción de café (Coffea arabica L.). En el municipio del Líbano, Tolima, Colombia. Colombia Forestal, 16(1), 31-21.

Arcila, J., Farfan, F., Moreno, A., Salazar, L., \& Hincapié, E. (2007). Sistemas de producción de café en Colombia. Cenicafé. https://biblioteca,cenicafe,org/bitstream/10778/720/1/Sistemas\%20producci\%C3\%B3n\%20caf\%C3\%A9\%20 Colombia.pdf

Aristizábal, A., \& Duque, O. (2006). Determinación de economías de escala en el proceso de beneficio del café en Colombia. Centro Nacional de Investigación de Café, 57(1), 17-30. https://www.cenicafe.org/es/publications/ $\operatorname{arc057\% 2801\% 29017-030.pdf~}$

Avelino, J., Barboza, B., Araya, J., Fonseca, C., Davrieux, F., Guyot, B., \& Cilas, C. (2005). Effects of slope exposure, altitude and yield on coffee quality in two altitude terroirs of Costa Rica, Orosí and Santa Maria de Dota. Journal of the Science of Food and Agriculture, 85(11), 1869-1876. https://doi.org/10.002/jsfa.2188

Banegas, K. (2009). Identificación de las fuentes de variación que tienen efecto sobre la calidad de café (Coffea arabica) en los municipios de El Paraíso y Alauca, Honduras (Tesis de Maestría, Centro Agronómico Tropical de Investigación y Enseñanza). Repositorio del Centro Agronómico Tropical de Investigación y Enseñanza. https://agritrop.cirad. fr/556129/1/document_556129.pdf

Bertrand, B., Vaast, P., Alpizar, E., Etienne, H., Davrieux, F., \& Charmetant, P. (2006) Comparison of bean biochemical composition and beverage quality of Arabica hybrids involving Sudanese-Ethiopian origins with traditional varieties at various elevations in Central America. Tree Physiology, 26(9), 1239-1248. https://doi.org/10.1093/treephys/26.9.1239

Bosselmann, A., Dons, T., Oberthur, C., Olsen, S., Raebild, A., \& Usma, H. (2009). The influence of shade trees on coffee quality in small holder coffee agroforestry systems in Southern Colombia. Agriculture, Ecosystems \& Environment, 129(103), 253-260. https://doi.org/10.1016/j.agee.2008.09.004

Buenaventura, S., \& Castaño, C. (2002). Influencia de la altitud en la calidad de bebida de muestras de café procedente del ecotopo 206B en Colombia. Revista Cenicafé, 53(2), 119 -131.

Burgos, A. (2015). Potencial del sector caficultor en Colombia, Nariño y la importancia de la responsabilidad social para su crecimiento económico [Tesis de Especialidad, Universidad Militar Nueva Granada]. Repositorio de la Universidad Militar de Nueva Granada. https://repository.unimilitar.edu.co/handle/10654/7816?locale=en

Chávez, D., Miranda, I., Varela, M., \& Fernández, L. (2010). Utilización del análisis de clúster con variables mixtas en la selección de genotipos de maíz (Zea mays L.). Revista Investigación Operacional, 30(3), 209-216. 
DaMatta, F., Ronchi, C., Maestri, M., \& Barros, R. (2007). Ecophysiolo $\neg$ gy of coffee growth and production. Brazilian Journal of Plant Physiology, 19(4), 485-510. https://doi.org/10.1590/S1677-04202007000400014

De La Rosa, M. (2011, abril 2). Nariño huele a cafés especiales. Portafolio. https://www.portafolio.co/negocios/empresas/ narino-huele-cafes-especiales-121486

Díaz, V., Pérez, J., Magallanes, R., Pinzón, E., Coss, M., \& Cabrera, M. (2014). Control biológico de la broca del café Hypothenemus hampei Ferrari (Coleóptera: Curculionidae) con diferentes dosis del hongo Beauveria bassiana (Balls) Vuill. (Moniliales: Moniliaceae) en la Unión Juárez, Chiapas, México. Vedalia, 15(1), 15-21.

Echavarría, J., Esguerra, P., Mcallister, D., \& Robayo, C. (2015). Misión de estudios para la competitividad de la caficultura en Colombia. Fondo Nacional del Café. https://www.urosario.edu.co/Mision-Cafetera/Archivos/Mision-de-estudiospara-competitividad-de-la-Cafic/

Federación Nacional de Cafeteros de Colombia. (2018). Informe de gestión 2018, rentabilidad- legitimidad y resiliencia. https:// federaciondecafeteros.org/app/uploads/2019/10/Informe_Gestion_2018_compressed-1.pdf

Federación Nacional de Cafeteros de Colombia. (2020). Aprenda a vender su café. https://federaciondecafeteros.org/wp/ servicios-al-caficultor/aprenda-a-vender-su-cafe/

Gamboa, R., Mosquera, S., \& Paz, 1. (2015). Caracterización física de café especial (Coffea arabica) en el municipio de Chachagüí. Revista Lasallista de Investigación, 12(1), 90-98. https://doi.org/10.22507/rli.v12n1a9

Geromel, C., Pires, F., Davrieux, F., \& Guyot, B. (2007). Effects of shade on the development and sugar metabolism of coffee (Coffea arabica L.). Plant Physiology and Biochemistry, 46(5-6), 569-579. https://doi.org/10.1016/j. plaphy.2008.02.006

Giovannucci, D., \& Koekoek, F. (2003). The state of sustainable coffee: a study of twelve major markets. International Institute for Sustainable Development. https://www.iisd.org/system/files/publications/trade_state_sustainable_coffee.pdf

Gómez, L., Caballero, A., \& Baldión, J. (1991). Ecotopos cafeteros de Colombia. Repositorio del Centro Nacional de Investigaciones de Café. https://biblioteca.cenicafe.org/bitstream/10778/818/1/lib13731.pdf

Jezeer, R, \& Verweij, P. (2015). Café en Sistema Agroforestal - doble dividendo para la biodiversidad y los pequeños agricultores en Perú. Docplayer. https://docplayer.es/22901991-Cafe-en-sistemas-agroforestales-doble-dividendopara-la-biodiversidad-y-los-pequenos-agricultores-en-peru.html

Joët, T., Laffargue, A., Descroix, F., Doulbeau, S., Bertrand, B., kochko, A., \& Dussert, S. (2010). Influence of environmental factors, wet processing and their interactions on the biochemical composition of green Arabica coffee beans. Food Chemistry, 118(3), 693-701. https://doi.org/10.1016/j.foodchem.2009.05.048

Lara, L., \& Vaast, P. (2007, September, 17-21). Effects of altitude, shade, yield and fertilization on coffee quality (Coffea arabica L. var. Caturra) produced in agroforestry systems of the Northern Central Zones of Nicaragua [Conference presentation]. $2^{\text {nd }}$ International Symposium on Multi-Strata Agroforestry Systems with Perennial Crop, Turrialba, Costa Rica. https://doi.org/10.13140/RG.2.1.4689.1289

Maurice, N., \& Davis, J. (2011). Unraveling the underlying causes of price volatility in world coffee and cocoa commodity markets. United Nations Conference on Trade and Development. https:/unctad.org/system/files/official-document/ suc-miscDP01_en.pdf 
McNougher, P. (2013). Insight special: Debunking coffee myths. Coffee division of ED\&F Man. https://www.yumpu.com/en/ document/read/22891202/insight-special-debunking-coffee-myths-bijdendijk

Ministerio de Agricultura y Desarrollo Rural. (2018). Reporte: Comparativo de área, producción rendimiento y participación departamental por cultivo. https://www.agronet.gov.co/estadistica/Paginas/home.aspx?cod=3

Montilla, J., Arcila, J., Aristizábal, M., Montoya, E., Puerta, G., Oliveros, C., \& Cadena, G. (2008). Caracterización de algunas propiedades de conversión del café durante el proceso de beneficio húmedo tradicional. Centro Nacional de Investigaciones de Café, 59(2), 120-142. https://www.cenicafe.org/es/publications/arc059(02)120-142.pdf

Montoya, E. (1999). Caracterización de la infestación del café por la broca y efecto del daño en la calidad de bebida. Centro Nacional de Investigaciones de Café, 50(4), 245-258. https://www.cenicafe.org/es/publications/arc050\%2804 \%29245-258.pdf

Organización Internacional del Café. (2019). Informe de la OIC sobre desarrollo cafetero del 2019 (ED 2318/19). http://www. ico.org/documents/cy2018-19/ed-2318c-overview-flagship-report.pdf

Orozco, N., Guacas, A., \& Bacca, T. (2011). Caracterización de fincas cafeteras por calidad de la bebida y algunas condiciones ambientales y agronómicas. Revista de Ciencias Agrícolas, 28(2), 9-17. http://sired.udenar.edu.co/835/

Otzen, T., \& Manterola, C. (2017). Técnicas de muestreo en un estudio poblacional. International Journal of Morphology, 35(1), 227-232.http://www.intjmorphol.com/wp-content/uploads/2017/04/art_37_351.pdf

Panhuysen, S., \& Pierrot, J. (2014). Barómetro de café 2014. Federación de Cafetaleros. https://federaciondecafeteros.org/static/ files/5Barometro_de_cafe2014.pdf

Pérez, E., Partida, J., \& Martínez, D. (2005). Determinación de las subdenominaciones de origen del Café Veracruz (estudio preliminar). Revista Geografía Agrícola, 35, 35-56. https://www.redalyc.org/pdf/757/75703502.pdf

Ponte, S. (2004). Standards and sustainability in the coffee sector: a global value chain approach. United Nations Conference on Trade and Development and the International Institute for Sustainable Development. https://www.researchgate. net/publication/264713468_Standards_and_Sustainability_in_the_Coffee_Sector_A_Global_Value_Chain_Approach

Puerta-Quintero, G. (2001). Cómo garantizar la buena calidad de la bebida del café y evitar los defectos (Avances técnicos 284). Centro Nacional de Investigaciones del Café. https://www.researchgate.net/publication/324066212_Como_ garantizar_la_buena_calidad_de_la_bebida_del_cafe_y_evitar_los_defectos

Puerta-Quintero, G. (2016). Calidad física del café de varias regiones de Colombia según altitud, suelos y buenas prácticas de beneficio. Revista del Centro Nacional de Investigaciones del Café, 67(1), 7-40. https://www.cenicafe.org/es/ publications/1.Calidad.pdf

Ramos, L., \& Criollo, H. (2017). Calidad física y sensorial de Coffea arabica L. variedad Colombia, perfil Nespresso AAA, La Unión Nariño. Revista de Ciencias Agrícolas, 34(2), 83-97. http://dx.doi.org/10.22267/rcia.173402.74

Ramírez, V., Jaramillo, A., \& Arcila, J. (2013). Factores climáticos que intervienen en la producción de café en Colombia. En S. M. María (Ed.), Manual del cafetero colombiano (Tomo I, pp. 205-238). Federación Nacional de Cafeteros de Colombia, \& Centro de Investigaciones en Café.

Rosas, J., Escamilla, E., \& Ruiz, O. (2008). Relación de los nutrimentos del suelo con las características físicas y sensoriales del café orgánico. Terra Latinoamericana, 26(4), 375-384. http://www.scielo.org.mx/pdf/tl/v26n4/v26n4a10.pdf 
Sánchez, J. (2015). Plan de manejo de café en el ámbito del Vraem. Agroaldia. https://docplayer.es/87138239-Plan-de-manejode-cafe-en-el-ambito-del-vraem.html

Soto, L., Perfecto, I., \& Caballero, J. (2002). Shade over coffee: its effects on berry borer, lveaf rust and spontaneous herbs in Chiapas, Mexico. Agroforestry Systems, 55, 37-45. https://doi.org/10.1023/A:1020266709570

Specialty Coffee Association of America. (2004). SCAA. ¿What is specialty coffee? https://scanews.coffee/2017/03/17/ what-is-specialty-coffee/\#: :text=The $\% 20$ SCAA $\% 20$ defines $\% 20$ specialty $\% 20$ coffee, aspect $\% 20$ grading $\% 20$ and $\% 20$ cupping\%20tests

Specialty Coffee Association of America. (2015). SCAA protocols, Cupping Specialty Coffee. https://www.scaa.org/PDF/ resources/cupping-protocols.pdf

Suárez, J., Rodríguez, E., \& Durán, E. (2015). Efecto de las condiciones de cultivo, las características químicas del suelo y el manejo del grano en los atributos sensoriales del café (Coffea arabica L.) en taza. Acta agronómica, 64(4), $342-348$. https://doi.org/10.15446/acag.v64n4.44641

Valencia, K. (2016). Competitividad de las exportaciones de café de Colombia, Guatemala y México hacia el mercado estadounidense (2001-2014). Ciencia Ergo Sum, 23(3), 239-253. http://www.redalyc.org/articulo.oa?id=10448076001

Velásquez, C., \& Trávez, M. (2019). Café especial, una alternativa para el sector cafetero en Colombia. Universidad EAFIT. https://repository.eafit.edu.co/bitstream/handle/10784/15236/Mateo_Travez_Camilo_Velasquez_2019. pdf? sequence $=2 \&$ isAllowed $=\mathrm{y}$

Viloria, J. (2007). Economía regional. Banco de la República. https://www.banrep.gov.co/docum/Lectura_finanzas/pdf/ DTSER-87.pdf 